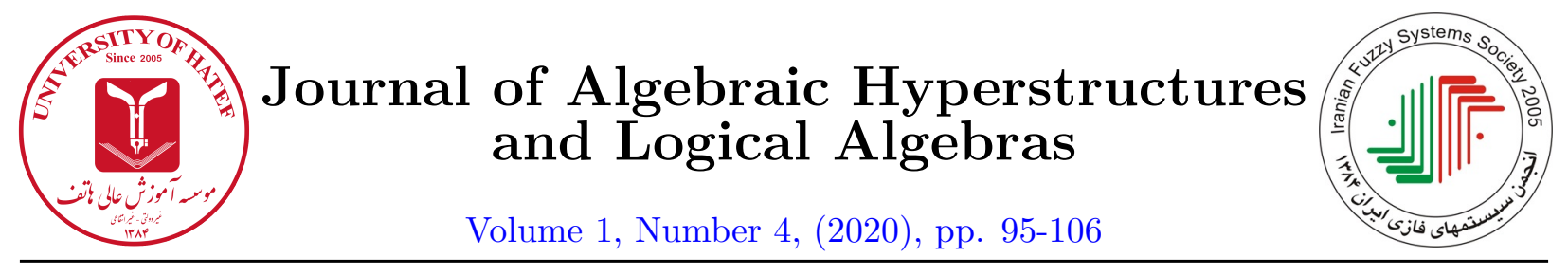

\title{
Relation between hyper $K$-algebras with superlattices and hypersemilattices
}

\author{
A. Rezazadeh ${ }^{1}$ and A. Radfar ${ }^{2}$ \\ ${ }^{1}$ Department of Mathematics, Maku Branch, Islamic Azad University, Maku, Iran \\ ${ }^{2}$ Department of Mathematics, Payame Noor University, p. o. box. 19395-3697, Tehran, Iran
}

Rezazade2008@gmail.com,radfaratefe@gmail.com

\begin{abstract}
In this paper, by considering the concepts of hypersemilattice and superlattice, we prove that any commutative and positive implicative hyper $K$-algebra, is a hypersemilattice. Moreover, we prove that any bounded commutative hyper $K$-algebra with some conditions, is a superlattice.
\end{abstract}

\section{Article Information}

Corresponding Author:

A. Rezazadeh;

Received: September 2020;

Revised: November 2020;

Accepted: November 2020,

Paper type: Original.

\section{Keywords:}

$B C K$-algebras, commutative hyper $K$-algebras, bounded hyper $K$-algebras, hypersemilattice, superlattice.

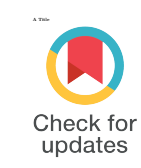

\section{$1 \quad$ Introduction}

The theory of hyperstructures was introduced in 1934 by Marty [6] at the 8th congress of Scandinavian Mathematicians. This theory has been subsequently developed by the contribution of various authors. Hyperstructures have many applications to several sectors of both pure and applied sciences. In [5], Jun et al, applied the hyperstructures to $B C K$-algebras and introduced the notion of a hyper $B C K$-algebra which is a generalization of $B C K$-algebra and investigated some related properties. In [3], Borzooei et al, defined the notions of hyper $I$-algebras and hyper $K$-algebras. Then they stated and proved some related theorems. Some basic definitions and propositions are found in [2], [10], [9] and [8].

https://doi.org/10.29252/HATEF.JAHLA.1.4.7 
Some researchers applied the hyperstructure to some accepts of lattice theory and the notion of hypersemilattice was introduced by Z. Bin et al. in [1] and the notion of superlattice was introduced by Mittas and Konstantinidou in [7].

In this paper, we provide some conditions for a hyper $K$-algebra to be a hypersemilattice. In follow, we introduce the notions $\wedge$ and $\vee$ on hyper $K$-algebras and we prove that every hyper $K$-algebra of order 3 by some conditions is a superlattice.

\section{Preliminaries}

In this section, we give some definitions and theorems that we need in the next sections.

Let $H$ be a non-empty set and $\circ$ a function from $H \times H$ to $\mathcal{P}(H)-\{\emptyset\}$, where $\mathcal{P}(H)$ denotes the power set of $H$. For two subsets $A$ and $B$ of $H$, denote by $A \circ B$ the set $\bigcup_{a \in A, b \in B} a \circ b$. We shall use $x \circ y$ instead of $x \circ\{y\},\{x\} \circ y$, or $\{x\} \circ\{y\}$.

Definition 2.1. [4] Let $L$ be a non-empty set endowed with hyperoperations $\wedge$ and $\vee$. Then $(L, \wedge, \vee)$ is called a hyperlattice if for any $x, y, z \in L$, the following conditions hold:

$(H L 1) x \in x \wedge x, x \in x \vee x$, $(H L 2) x \wedge y=y \wedge x, x \vee y=y \vee x$, $(H L 3)(x \wedge y) \wedge z=x \wedge(y \wedge z)$ $(H L 4) x \in x \wedge(x \vee y), x \in x \vee(x \wedge y)$.

Definition 2.2. [1] Let $L$ be a non-empty set with a binary hyperoperation $\circ$ on $L$ such that for all $x, y, z \in L$, the following conditions hold:

(i) $x \in x \circ x$,

(ii) $x \circ y=y \circ x$,

(iii) $(x \circ y) \circ z=x \circ(y \circ z)$.

Then $(L, \circ)$ is called a hypersemilattice.

Definition 2.3. [7] A superlattice is a partially ordered set $(S,<)$ with two hyperoperations $\vee$ and $\wedge$ such that for all $x, y, z \in S$, the following properties hold:

(S1) $x \in x \vee x$ and $x \in x \wedge x$,

(S2) $x \vee y=y \vee x$ and $x \wedge y=y \wedge x$,

(S3) $(x \vee y) \vee z=x \vee(y \vee z)$ and $(x \wedge y) \wedge z=x \wedge(y \wedge z)$,

(S4) $x \in x \vee(x \wedge y)$ and $x \in x \wedge(x \vee y)$,

(S5) if $x<y$, then $y \in x \vee y$ and $x \in x \wedge y$,

(S6) $y \in x \vee y$ or $x \in x \wedge y$ implies $x<y$.

Definition 2.4. [3] By a hyper K-algebra we mean a non-empty set $H$ endowed with a hyperoperation "०" and a constant 0 that for all $x, y, z \in H$, it satisfies in the following axioms:

$(H K 1)(x \circ z) \circ(y \circ z)<x \circ y$,

$(H K 2)(x \circ y) \circ z=(x \circ z) \circ y$,

(HK3) $x<x$,

(HK4) $x<y$ and $y<x$ imply $x=y$,

(HK5) $0<x$,

where $x<y$ is defined by $0 \in x \circ y$ and for every $A, B \subseteq H, A<B$ is defined by $\exists a \in A, \exists b \in B$ such that $a<b$.

Definition 2.5. [3] Let $(H, \circ, 0)$ be a hyper $K$-algebra. Then $(H, \circ, 0)$ is said to be a commutative hyper $K$-algebra, if for all $x, y \in H$,

$$
x \circ(x \circ y)=y \circ(y \circ x) .
$$


Definition 2.6. [3] Let $(H, \circ, 0)$ be a hyper $K$-algebra. Then $(H, \circ, 0)$ is said to be a positive implicative hyper $K$-algebra, if for all $x, y, z \in H$,

$$
(x \circ y) \circ z=(x \circ z) \circ(y \circ z) .
$$

Definition 2.7. [3] Let $(H, \circ, 0)$ be a hyper $K$-algebra. Then $(H, \circ, 0)$ is said to be a bounded hyper $K$-algebra, if there exists an element 1 such that $x<1$, for all $x \in H$.

Theorem 2.8. [3] Let $H$ be a hyper $K$-algebra. Then for all $x, y, z \in H$ and $A, B \subseteq H$, the following statements hold:

(i) $x \in x \circ 0$,

(ii) $x \circ y<z \Leftrightarrow x \circ z<y$,

(iii) $(x \circ z) \circ(x \circ y)<y \circ z$,

(iv) $x \circ(x \circ y)<y$,

(v) $x \circ y<x$,

(vi) $A \circ B<A$,

(vii) $A \subseteq B \Rightarrow A<B$,

(viii) $(A \circ C) \circ(A \circ B)<B \circ C$,

(ix) $A \circ B<C \Leftrightarrow A \circ C<B$.

\section{Relation between hyper $K$-algebras and hypersemilattices}

In this section, we prove that every commutative and positive implicative hyper $K$-algebra is a hypersemilattice.

Definition 3.1. In any commutative hyper $K$-algebra, for all $x, y \in H$, we denote

$$
x \cap y=\{z \mid z \in y \circ(y \circ x)\} .
$$

Proposition 3.2. Let $H$ be a commutative hyper $K$-algebra. Then for all $x, y \in H$, the following properties hold:

(i) $x \cap y<x$ and $x \cap y<y$,

(ii) $x \cap y=y \cap x$,

(iii) $x \in x \cap x$,

(iv) If $x<y$, then $x \in x \cap y$.

Proof. (i) Let $x, y \in H$. By Theorem $[.8($ iii $)$ and (v), $x \circ(x \circ y)<x$ and $x \circ(x \circ y)<y$. Hence $x \cap y<x$ and $x \cap y<y$.

(ii) The proof is straightforward.

(iii) Since $0 \in x \circ x$, we get $x \circ 0 \subseteq x \circ(x \circ x)$. Then by Theorem $[2.8(i), x \in x \circ 0$. Hence, $x \in x \circ(x \circ x)$, and so $x \in x \cap x$.

(iv) Let $x<y$, for $x, y \in H$. Then $0 \in x \circ y$ and so $x \circ 0 \subseteq x \circ(x \circ y)$. Thus by Theorem [2.8(i), $x \in x \circ 0$, and so $x \in x \circ(x \circ y)$. Hence, $x \in y \cap x=x \cap y$.

Theorem 3.3. Let $H$ be a commutative and positive implicative hyper K-algebra. Then for all $x, y, z \in H$,

$$
(x \cap y) \cap z=x \cap(y \cap z) .
$$


Proof. Let $x, y, z \in H$. Then

$$
\begin{aligned}
(x \cap y) \cap z= & \bigcup\{u \cap z \mid u \in x \cap y\} \\
& =\bigcup\{u \circ(u \circ z) \mid u \in y \circ(y \circ x)\} \\
& =(y \circ(y \circ x)) \circ((y \circ(y \circ x)) \circ z) \\
& =(y \circ(y \circ x)) \circ((y \circ z) \circ(y \circ x))(\text { by }(\text { HK2 })) \\
& \\
= & (y \circ(y \circ z)) \circ(y \circ x)(\text { by Definition 2.6) } \\
= & (y \circ(y \circ x)) \circ(y \circ z)(\text { by }(\text { HK2 })) \\
= & (y \circ(y \circ z)) \circ((y \circ x) \circ(y \circ z))(\text { by Definition 2.6 }) \\
= & (y \circ(y \circ z)) \circ((y \circ(y \circ z)) \circ x)(\text { by }(\text { HK2 })) \\
= & \bigcup\{t \circ(t \circ x) \mid t \in y \circ(y \circ z)\} \\
= & \bigcup\{x \cap t \mid t \in y \cap z\} \\
= & x \cap(y \cap z) .
\end{aligned}
$$

Corollary 3.4. Let $(H, \circ)$ be a commutative and positive implicative hyper $K$-algebra. Then $(H, \cap)$ is a hypersemilattice.

Proof. By Definition [2.2, Proposition $[2.2$ and Theorem 3.3 , the proof is obvious.

Example 3.5. Let $H=\{0, a, b\}$ and the hyperoperation" $\circ "$ on $H$ defined as follows:

\begin{tabular}{c|ccc}
$\circ$ & 0 & $a$ & $b$ \\
\hline 0 & $\{0\}$ & $\{0, a\}$ & $\{0, a, b\}$ \\
$a$ & $\{a\}$ & $\{0, a\}$ & $\{0, a, b\}$ \\
$b$ & $\{b\}$ & $\{b\}$ & $\{0, a, b\}$
\end{tabular}

Then $(H, \circ, 0)$ is a commutative positive implicative hyper K-algebra. Hence by Corollary B.4, $(H, \cap)$ is a hypersemilattice.

\section{Relation between hyper $K$-algebras and superlattices}

In this section, we introduce some operations such as $\wedge$ and $\vee$ on hyper $K$-algebra and investigate some properties and relation between them. Then we prove that any bounded commutative hyper $K$-algebra with some conditions $L$ is a superlattice.

Definition 4.1. Let $(H, \circ, 0)$ be a hyper $K$-algebra. Then $(H, \circ, 0)$ is said to be a complemented hyper $K$-algebra, if $H$ is bounded and $1 \circ x$ has a least element with respect to $<$, for all $x \in H$.

We note that if $H$ is bounded, then by $(H K 4)$ we can easily get that 1 is unique. Also, if $H$ is complemented, then we use $x^{\prime}$ to denote $\min (1 \circ x)$. 
Example 4.2. Let $H=\{0, a, b, 1\}$ and hyperoperations " $\circ_{1} "$ and $" \circ_{2} "$ on $H$ defined as follows:

\begin{tabular}{l|llll}
$\circ_{1}$ & 0 & $a$ & $b$ & 1 \\
\hline 0 & $H$ & $H$ & $H$ & $H$ \\
$a$ & $\{a\}$ & $H$ & $\{a, b, 1\}$ & $H$ \\
$b$ & $\{b\}$ & $\{a, b, 1\}$ & $H$ & $H$ \\
1 & $\{1\}$ & $\{a, b\}$ & $\{a\}$ & $H$
\end{tabular}

\begin{tabular}{l|llll}
$\circ_{2}$ & 0 & $a$ & $b$ & 1 \\
\hline 0 & $H$ & $H$ & $H$ & $H$ \\
$a$ & $\{a\}$ & $H$ & $\{a, b, 1\}$ & $H$ \\
$b$ & $\{b\}$ & $\{a, b, 1\}$ & $H$ & $H$ \\
1 & $\{1\}$ & $\{a, b, 1\}$ & $\{a, 1\}$ & $H$
\end{tabular}

Then $\left(H, \circ_{2}\right)$ is a bounded commutative hyper $K$-algebra. But $\left(H, \circ_{1}\right)$ is not commutative, because

$$
H=a \circ_{2} H=a \circ_{1}\left(a \circ_{1} 1\right) \neq 1 \circ_{1}\left(1 \circ_{1} a\right)=1 \circ_{1}\{a, b\}=\{a, b\} .
$$

We can see $(H, \circ)$ in Example 3.5 is complemented but $\left(H, \circ_{1}\right)$ and $\left(H, \circ_{2}\right)$ are not complemented, since $\min (1 \circ a)$ does not exist in both of them.

Definition 4.3. In any commutative hyper $K$-algebra, for all $x, y \in H$, we define

$$
x \wedge y=\{z \mid z \in y \circ(y \circ x) \text { such that } z<x \text { and } z<y\} .
$$

By $A \wedge B$ we mean $\bigcup_{a \in A, b \in B} a \wedge b$.

Proposition 4.4. Let $H$ be a bounded commutative hyper $K$-algebra. Then for all $x, y \in H$, the following properties hold:

(i) $x \in x \wedge x, x \in x \wedge 1,1 \in 1 \wedge 1$ and $0 \wedge 1=0$,

(ii) $x \wedge y<x, x \wedge y<y$ and $x \wedge y=y \wedge x$,

(iii) $x \wedge 0=0 \wedge x=\{0\}$,

(iv) If $x<y$, then $x \in x \wedge y$,

(v) If $x \neq 1$, then $1 \notin x \wedge 1$ and $1 \notin x \wedge x$,

(vi) If $x \wedge 1=\{x\}$, then $y \in x \circ 1$ implies $x \nless y$,

(vii) If $x \wedge 1=\{x\}$, then $x, 1 \notin x \circ 1$,

(viii) If $x \wedge 1=\{x\}$, then $0,1 \notin 1 \circ x$,

(ix) If $H$ is a chain respect to $<$, then $x \wedge y \neq \emptyset$,

( $x$ ) If $H$ is of order less than 5 , then $x \wedge y \neq \emptyset$.

Proof. ( $i$ ) By $(H K 3), 0 \in x \circ x$. Hence $x \circ 0 \subseteq x \circ(x \circ x)$. By Theorem $[2.8(\mathrm{i}), x \in x \circ 0$ and so $x \in x \circ(x \circ x)$. Since $x<x$, we get $x \in x \wedge x$. By the similar way we can prove $x \in x \wedge 1,1 \in 1 \wedge 1$ and $0 \in 0 \wedge 1$.

(ii) By definition of $\wedge$, the proof is obvious.

(iii) By (HK3), $0 \in x \circ x$ and by Theorem [2.8(i), $0 \in x \circ x \subseteq x \circ(x \circ 0)$. Thus $0 \in x \wedge 0$. If $y \in x \wedge 0$, then $y<0$. By $(H K 5), 0<y$ and by $(H K 4)$, we have $y=0$. Hence $x \wedge 0=0 \wedge x=\{0\}$.

(iv) Let $x<y$ for $x, y \in H$. Then $0 \in x \circ y$ and so $x \circ 0 \subseteq x \circ(x \circ y)$. By Theorem [2.8(i), $x \in x \circ 0$ and so $x \in x \circ(x \circ y)$. Since $x<y$, we get $x \in y \wedge x=x \wedge y$.

$(v)$ If $x \neq 1$, then $x<1$, for all $x \in H$. By $(H K 4), 1 \nless x$. By Definition [2.], $1 \notin x \wedge 1$. By the similar way, we have $1 \notin x \wedge x$.

(vi) Let $x \wedge 1=\{x\}$. Suppose $x<y$. If $y \in x \circ 1$, then $0 \in x \circ y \subseteq x \circ(x \circ 1)$. Since $0<1$ and $0<x$, we get $0 \in x \wedge 1=\{x\}$, which is a contradiction. Hence, if $y \in x \circ 1$, then $x \nless y$.

(vii) Since $x<x$ and $x<1$, by (vi), we obtain $x, 1 \notin x \circ 1$. 
(viii) Let $x \wedge 1=\{x\}$. If $1 \in 1 \circ x$, then $0 \in 1 \circ 1 \subseteq 1 \circ(1 \circ x)$. Since $0<1$ and $0<x$, we consequence $0 \in x \wedge 1=\{x\}$, which is a contradiction. Hence $1 \notin 1 \circ x$. Also, since $1 \nless x$, we get $0 \notin 1 \circ x$. Therefore, $0,1 \notin 1 \circ x$.

(ix) By $(i v)$ the proof is clear.

$(x)$ Every bounded hyper $K$-algebra of order 2 and 3 is a chain and so by $(i x)$ we imply $x \wedge y \neq \emptyset$ , for any $x, y \in H$. Now, let $H=\{0, a, b, 1\}$ be a bounded commutative hyper $K$-algebra. Then by $(i),(i i)$ and (iii), it is sufficient to prove $a \wedge b \neq \emptyset$. If $a<b$ or $b<a$, then by (iv) we get $a \wedge b \neq \emptyset$. Now, suppose $a \nless b$ and $b \nless a$. Then $0 \notin(a \circ b) \cap(b \circ a)$. Since $a \circ b<a$, there exists $u \in a \circ b$ such that $u<a$. We note $0 \notin a \circ b, 1 \nless a$ and $b \nless a$. Thus $u \neq 0,1, b$ and so $u=a$. Hence $a \in a \circ b$. Thus $0 \in a \circ a \subseteq a \circ(a \circ b)$ and so $0 \in a \wedge b$. Therefore, $a \wedge b \neq \emptyset$.

Proposition 4.5. Let $H$ be a commutative hyper $K$-algebra. If $(x \wedge y) \wedge z=x \wedge(y \wedge z)$, for all $x, y, z \in X$, then $x \wedge y$ is the greatest lower bound of $x$ and $y$.

Proof. By Proposition $4.4(i i), x \wedge y<x$ and $x \wedge y<y$ for all $x, y \in H$. Now, let $z<x$ and $z<y$, for some $x, y, z \in H$. By Proposition $4.4(i v), z \in z \wedge x$ and $z \in z \wedge y$. By assumption,

$$
z \in z \wedge x \subseteq(z \wedge y) \wedge x=z \wedge(y \wedge x)=z \wedge(x \wedge y) .
$$

Thus there exists $t \in x \wedge y$ such that $z \in z \wedge t$ and so $z<t$. Therefore, $z<x \wedge y$.

Example 4.6. In Examples 3.5 and 4.2 , we can see $(H, \circ)$ and $\left(H, \circ_{2}\right)$ are bounded commutative hyper K-algebras. By easy calculations we get

\begin{tabular}{l|lll}
$\wedge$ & 0 & $a$ & $b$ \\
\hline 0 & $\{0\}$ & $\{0\}$ & $\{0\}$ \\
$a$ & $\{0\}$ & $\{0, a\}$ & $\{0, a\}$ \\
$b$ & $\{0\}$ & $\{0, a\}$ & $\{0, a, b\}$
\end{tabular}

\begin{tabular}{l|llll}
$\wedge_{2}$ & 0 & $a$ & $b$ & 1 \\
\hline 0 & $\{0\}$ & $\{0\}$ & $\{0\}$ & $\{0\}$ \\
$a$ & $\{0\}$ & $\{0, a\}$ & $\{0\}$ & $\{0, a\}$ \\
$b$ & $\{0\}$ & $\{0\}$ & $\{0, b\}$ & $\{0, b\}$ \\
1 & $\{0\}$ & $\{0, a\}$ & $\{0, b\}$ & $\{0, a, b, 1\}$
\end{tabular}

We can see that, above hyperoperations are associative.

The following example shows that the converse of Proposition 4.5 is not correct, in general.

Example 4.7. Let $H=\{0, a, b, 1\}$ and the hyperoperation" $\circ "$ on $H$ defined as follows:

\begin{tabular}{c|cccc}
$\circ$ & 0 & $a$ & $b$ & 1 \\
\hline 0 & $\{0, a, b, 1\}$ & $\{0, a, b, 1\}$ & $\{0\}$ & $\{0\}$ \\
$a$ & $\{a, b, 1\}$ & $\{0, a, b, 1\}$ & $\{0, a\}$ & $\{0\}$ \\
$b$ & $\{b\}$ & $\{b\}$ & $\{0, a, b, 1\}$ & $\{0, a, b, 1\}$ \\
1 & $\{1\}$ & $\{b\}$ & $\{a, b, 1\}$ & $\{0, a, b, 1\}$
\end{tabular}

Then $(H, \circ, 0)$ is a commutative hyper $K$-algebra. By routine calculations we get

\begin{tabular}{c|cccc}
$\wedge$ & 0 & $a$ & $b$ & 1 \\
\hline 0 & $\{0\}$ & $\{0\}$ & $\{0\}$ & $\{0\}$ \\
$a$ & $\{0\}$ & $\{0, a\}$ & $\{0, a, b\}$ & $\{a\}$ \\
$b$ & $\{0\}$ & $\{0, a, b\}$ & $\{0, a, b\}$ & $\{0, a, b\}$ \\
1 & $\{0\}$ & $\{a\}$ & $\{0, a, b\}$ & $\{0, a, b, 1\}$
\end{tabular}


We can see that for all $x, y \in H, x \wedge y$ is the greatest lower bound of $x$ and $y$ but $\wedge$ is not associative operator. Because

$$
a \wedge(1 \wedge 1)=a \wedge\{0, a, b, 1\}=\{0, a, b\} \neq\{a\}=\{a\} \wedge 1=(a \wedge 1) \wedge 1 .
$$

Proposition 4.8. Let $H$ be a bounded commutative hyper $K$-algebra. Then the following properties hold:

(i) If $x, y$ or $z$ equal to 0 , then $(x \wedge y) \wedge z=x \wedge(y \wedge z)$,

(ii) $(x \wedge x) \wedge x=x \wedge(x \wedge x)$,

(iii) If $x \wedge x=\{x\}$ or $\{0, x\}$ and $x \wedge y=\{x\}$ or $\{0, x\}$, then $(x \wedge x) \wedge y=x \wedge(x \wedge y)$,

(iv) If $x \wedge 1=\{0, x\}$ and $1 \wedge 1=\{1\}$ or $\{0,1\}$, then $(x \wedge 1) \wedge 1=x \wedge(1 \wedge 1)$,

(v) If $x \wedge 1=\{0, x\}, 1 \wedge 1=\{1, x\}$ or $\{0, x, 1\}$ and $x \wedge x=\{x\}$ or $\{0, x\}$, then $(x \wedge 1) \wedge 1=x \wedge(1 \wedge 1)$.

Proof. The proof of $(i)$ and (ii), by Proposition $4.4(i i i)$ and (ii), is clear.

(iii) We have four cases:

Case 1. Let $x \wedge x=x \wedge y=\{x\}$. Then $(x \wedge x) \wedge y=\{x\} \wedge y=\{x\}$ and $x \wedge(x \wedge y)=x \wedge\{x\}=\{x\}$. Hence $(x \wedge x) \wedge y=x \wedge(x \wedge y)$.

Case 2. Let $x \wedge x=\{0, x\}$ and $x \wedge y=\{x\}$. Then $(x \wedge x) \wedge y=\{0, x\} \wedge y=\{0, x\}$ and $x \wedge(x \wedge y)=x \wedge\{x\}=\{0, x\}$. Hence $(x \wedge x) \wedge y=x \wedge(x \wedge y)$.

Case 3. Let $x \wedge x=\{x\}$ and $x \wedge y=\{0, x\}$. Then $(x \wedge x) \wedge y=\{x\} \wedge y=\{0, x\}$ and $x \wedge(x \wedge y)=x \wedge\{0, x\}=\{0, x\}$. Hence $(x \wedge x) \wedge y=x \wedge(x \wedge y)$.

Case 4. Let $x \wedge x=x \wedge y=\{0, x\}$. Then $(x \wedge x) \wedge y=\{0, x\} \wedge y=\{0, x\}$ and $x \wedge(x \wedge y)=$ $x \wedge\{0, x\}=\{0, x\}$. Hence $(x \wedge x) \wedge y=x \wedge(x \wedge y)$.

(iv) Let $x \wedge 1=\{0, x\}$. Then $(x \wedge 1) \wedge 1=\{0, x\} \wedge 1=\{0, x\}$. Thus by assumption, we have two cases:

Case 1. If $1 \wedge 1=\{1\}$, then $x \wedge(1 \wedge 1)=x \wedge\{1\}=\{0, x\}$. Hence $(x \wedge 1) \wedge 1=x \wedge(1 \wedge 1)$.

Case 2. If $1 \wedge 1=\{0,1\}$, then $x \wedge(1 \wedge 1)=x \wedge\{0,1\}=\{0, x\}$. Hence $(x \wedge 1) \wedge 1=x \wedge(1 \wedge 1)$.

$(v)$ If $x \wedge 1=\{0, x\}$, then $(x \wedge 1) \wedge 1=\{0, x\} \wedge 1=\{0, x\}$.

If $1 \wedge 1=\{1, x\}$, then $x \wedge(1 \wedge 1)=x \wedge\{1, x\}=x \wedge 1 \cup x \wedge x=\{0, x\}$. Hence $(x \wedge 1) \wedge 1=x \wedge(1 \wedge 1)$.

If $1 \wedge 1=\{0, x, 1\}$, then $x \wedge(1 \wedge 1)=x \wedge\{0, x, 1\}=\{0, x\}$. Hence $(x \wedge 1) \wedge 1=x \wedge(1 \wedge 1)$.

Definition 4.9. Let $H$ be a bounded commutative complemented hyper $K$-algebra. We say $H$ satisfies in conditions $(L)$, if for all $x, y \in H$, the following conditions hold:

$\left(L_{1}\right) x \wedge y \neq \emptyset$,

$\left(L_{2}\right) x^{\prime} \circ y=y^{\prime} \circ x$

$\left(L_{3}\right)\left(x^{\prime}\right)^{\prime}=x$.

In the next example, we show that there exists a bounded commutative complemented hyper $K$-algebra with condition $(L)$. Also, we show that the conditions $\left(L_{2}\right)$ and $\left(L_{3}\right)$ are independent.

Example 4.10. (i) Let $H=\{0, a, 1\}$ and the hyperoperation" $\circ "$ on $H$ defined as follows:

\begin{tabular}{c|ccc}
$\circ$ & 0 & $a$ & 1 \\
\hline 0 & $\{0\}$ & $\{0, a, 1\}$ & $\{0\}$ \\
$a$ & $\{a, 1\}$ & $\{0, a\}$ & $\{0, a, 1\}$ \\
1 & $\{1\}$ & $\{a, 1\}$ & $\{0\}$
\end{tabular}

Then $(H, \circ, 0)$ is a bounded commutative hyper $K$-algebra and satisfies in condition $(L)$.

(ii) Let $H=\{0, a, 1\}$ and the hyperoperation " $\circ$ " on $H$ defined as follows: 


\begin{tabular}{c|ccc}
$\circ$ & 0 & $a$ & 1 \\
\hline 0 & $\{0, a\}$ & $\{0, a, 1\}$ & $\{0,1\}$ \\
$a$ & $\{a, 1\}$ & $\{0, a, 1\}$ & $\{0, a\}$ \\
1 & $\{1\}$ & $\{1\}$ & $\{0, a, 1\}$
\end{tabular}

Then $(H, \circ, 0)$ is a bounded commutative hyper $K$-algebra and $\min (1 \circ x)$ exist, for all $x \in H$. Since $a^{\prime}=\min (1 \circ a)=\min \{1\}=1$ and $\left(a^{\prime}\right)^{\prime}=\min (1 \circ 1)=\min \{0, a, 1\}=0 \neq a$, then $H$ does not satisfy in condition $\left(L_{3}\right)$. Also, $H$ does not satisfiy in condition $\left(L_{2}\right)$. Because

$$
1^{\prime} \circ 0=0 \circ 0=\{0, a\} \neq\{0, a, 1\}=1 \circ 1=0^{\prime} \circ 1 .
$$

(iii) Let $H=\{0, a, 1\}$ and the hyperoperation" $\circ "$ on $H$ defined as follows:

\begin{tabular}{c|ccc}
$\circ$ & 0 & $a$ & 1 \\
\hline 0 & $\{0,1\}$ & $\{0, a\}$ & $\{0,1\}$ \\
$a$ & $\{a, 1\}$ & $\{0, a, 1\}$ & $\{0, a, 1\}$ \\
1 & $\{1\}$ & $\{a, 1\}$ & $\{0,1\}$
\end{tabular}

Then $(H, \circ, 0)$ is a bounded commutative hyper $K$-algebra and $\min \{1 \circ x\}$ exist, for all $x \in H$. Also, $H$ satisfies in condition $\left(L_{3}\right)$. Since $1^{\prime} \circ a=0 \circ a=\{0, a\}$ and $a^{\prime} \circ 1=a \circ 1=\{0, a, 1\}$, we get $\left(L_{2}\right)$ does not hold.

(iv) Let $H=\{0, a, 1\}$ and the hyperoperation" $\circ "$ on $H$ defined as follows:

\begin{tabular}{c|ccc}
$\circ$ & 0 & $a$ & 1 \\
\hline 0 & $\{0, a, 1\}$ & $\{0, a, 1\}$ & $\{0, a, 1\}$ \\
$a$ & $\{a, 1\}$ & $\{0, a, 1\}$ & $\{0, a, 1\}$ \\
1 & $\{1\}$ & $\{1\}$ & $\{0, a, 1\}$
\end{tabular}

Then $(H, \circ, 0)$ is a bounded commutative hyper $K$-algebra and $\min (1 \circ x)$ exist, for all $x \in H$. Also, $H$ satisfies in condition $\left(L_{2}\right)$. Since $a^{\prime}=\min (1 \circ a)=\min \{1\}=1$ and $\left(a^{\prime}\right)^{\prime}=1^{\prime}=\min (1 \circ 1)=$ $\min \{0, a, 1\}=0$, we obtain $\left(L_{3}\right)$ does not hold.

Lemma 4.11. Let $H$ satisfies in condition $(L)$. Then the following statements hold:

(i) $0^{\prime}=1$ and $1^{\prime}=0$,

(ii) $1 \circ 0=\{1\}$,

(iii) $0 \circ 0=1 \circ 1$,

(iv) If $x^{\prime}=x$, for some $x \in H$, then $0 \circ x=x \circ 1, x \circ 0=1 \circ x$.

Proof. $(i)$ Since $0 \in 1 \circ 1$, we get $1^{\prime}=\min (1 \circ 1)=0$. Then by $\left(L_{3}\right)$, we have $0^{\prime}=\left(1^{\prime}\right)^{\prime}=1$.

(ii) By $(i)$, we get $0^{\prime}=1$ and so $\min (1 \circ 0)=1$. Since $x \leq 1$, for all $x \in H$, it is clear that $1 \circ 0=\{1\}$.

(iii), (iv) By $(i)$ and $\left(L_{2}\right)$, the proof is clear.

Notation. In any hyper $K$-algebras with condition $(L)$, for all $x, y \in H$, we define

$$
x \vee y=\left\{z \mid z \in\left(x^{\prime} \wedge y^{\prime}\right)^{\prime}\right\} .
$$

By $A \vee B$ we mean $\bigcup_{a \in A, b \in B} a \vee b$. 
Proposition 4.12. Let $H$ be a hyper $K$-algebra with condition $(L)$. Then for all $x, y \in H$ the following statements hold:

(i) $x<x \vee y$ and $y<x \vee y$,

(ii) $x \in x \vee x$,

(iii) $x \vee y=y \vee x$,

(iv) If $x<y$, then $y \in x \vee y$,

(v) If $x \in x \wedge y$ or $y \in x \vee y$, then $x<y$.

Proof. (i) By Proposition $4.4(i i), x^{\prime} \wedge y^{\prime}<x^{\prime}$, for all $x, y \in H$. Hence, there exists $u \in x^{\prime} \wedge y^{\prime}$ such that $u<x^{\prime}$. Then by $\left(L_{2}\right),\left(x^{\prime}\right)^{\prime} \circ u^{\prime}=\left(u^{\prime}\right)^{\prime} \circ x^{\prime}=u \circ x^{\prime}$. Since $0 \in\left(x^{\prime}\right)^{\prime} \circ u^{\prime}$, we get $\left(x^{\prime}\right)^{\prime}<u^{\prime}$, and so $x<u^{\prime} \in\left(x^{\prime} \wedge y^{\prime}\right)^{\prime}$. Hence, $x<x \vee y$. By the similar way, we can prove $y<x \vee y$.

(ii) By Proposition $4.4(i), x^{\prime} \in x^{\prime} \wedge x^{\prime}$. Thus $\left(x^{\prime}\right)^{\prime} \subseteq\left(x^{\prime} \wedge x^{\prime}\right)^{\prime}$, and so $x \in x \vee x$.

(iii) By definition of $\mathrm{V}$ and since $H$ is commutative, the proof is clear.

(iv) Let $x<y$, for some $x, y \in H$. Then by $\left(L_{2}\right), y^{\prime} \circ x^{\prime}=\left(x^{\prime}\right)^{\prime} \circ y=x \circ y$. Since $0 \in x \circ y$, we have $0 \in y^{\prime} \circ x^{\prime}$, and so $y^{\prime}<x^{\prime}$. By Proposition $4.4(i v), y^{\prime} \in x^{\prime} \wedge y^{\prime}$. Hence, $\left(y^{\prime}\right)^{\prime} \subseteq\left(x^{\prime} \wedge y^{\prime}\right)^{\prime}$. Therefore, $y \in x \vee y$.

(v) Let $x \in x \wedge y$, for some $x, y \in H$. By Definition 4.3, $x<y$. Now, let $y \in x \vee y$. Then $y \in\left(x^{\prime} \wedge y^{\prime}\right)^{\prime}$ and so $y^{\prime} \subseteq\left(\left(x^{\prime} \wedge y^{\prime}\right)^{\prime}\right)^{\prime}=x^{\prime} \wedge y^{\prime}$. By Definition 4.3, $y^{\prime}<x^{\prime}$ and so $\left(x^{\prime}\right)^{\prime}<\left(y^{\prime}\right)^{\prime}$. Therefore, $x<y$.

Proposition 4.13. Let $H$ be a hyper $K$-algebra with condition $(L)$. For any $x, y, z \in H,(x \vee y)^{\prime}=$ $x^{\prime} \wedge y^{\prime}$.

Proof. Let $u \in(x \vee y)^{\prime}$, for some $x, y \in H$. Then $u^{\prime} \subseteq x \vee y$, thus $u^{\prime} \in\left(x^{\prime} \wedge y^{\prime}\right)^{\prime}$, and so $u=\left(u^{\prime}\right)^{\prime} \subseteq\left(\left(x^{\prime} \wedge y^{\prime}\right)^{\prime}\right)^{\prime}$. Hence $u \in x^{\prime} \wedge y^{\prime}$. Therefore, $(x \vee y)^{\prime} \subseteq x^{\prime} \wedge y^{\prime}$. Now, suppose $t \in x^{\prime} \wedge y^{\prime}$. Then $t^{\prime} \subseteq\left(x^{\prime} \wedge y^{\prime}\right)^{\prime}$ and so $t^{\prime} \in x \vee y$. Thus $t=\left(t^{\prime}\right)^{\prime} \subseteq(x \vee y)^{\prime}$. Hence, $x^{\prime} \wedge y^{\prime} \subseteq(x \vee y)^{\prime}$, and so $(x \vee y)^{\prime}=x^{\prime} \wedge y^{\prime}$.

Theorem 4.14. Let $H$ be a hyper $K$-algebra with condition $(L)$. For any $x, y, z \in H$,

$$
(x \wedge y) \wedge z=x \wedge(y \wedge z) \text { if and only if }(x \vee y) \vee z=x \vee(y \vee z) \text {. }
$$

Proof. Let $(x \wedge y) \wedge z=x \wedge(y \wedge z)$ for all $x, y, z \in H$. Then

$$
\begin{aligned}
(x \vee y) \vee z & =\bigcup\{u \vee z \mid u \in x \vee y\} \\
& =\bigcup\left\{\left(u^{\prime} \wedge z^{\prime}\right)^{\prime} \mid u \in x \vee y\right\} \\
& =\bigcup\left\{\left(u^{\prime} \wedge z^{\prime}\right)^{\prime} \mid u^{\prime} \in(x \vee y)^{\prime}\right\} \\
& =\bigcup\left\{\left(u^{\prime} \wedge z^{\prime}\right)^{\prime} \mid u^{\prime} \in x^{\prime} \wedge y^{\prime}\right\} \text { (by Proposition 4.13) } \\
& =\left(\left(x^{\prime} \wedge y^{\prime}\right) \wedge z^{\prime}\right)^{\prime} \\
& =\left(x^{\prime} \wedge\left(y^{\prime} \wedge z^{\prime}\right)\right)^{\prime} \\
& =\left(x^{\prime} \wedge(y \vee z)^{\prime}\right)^{\prime}(\text { by Proposition 4.13) } \\
& =\bigcup\left\{\left(x^{\prime} \wedge t^{\prime}\right)^{\prime} \mid t \in y \vee z\right\} \\
& =\bigcup\{x \vee t \mid t \in y \vee z\} \\
& =x \vee(y \vee z)
\end{aligned}
$$

Conversely, let $(x \vee y) \vee z=x \vee(y \vee z)$ for all $x, y, z \in H$. By the similar way, we can prove that $(x \wedge y) \wedge z=x \wedge(y \wedge z)$. 
Theorem 4.15. Let $H$ be a hyper $K$-algebra with condition $(L)$ and $\wedge$ be associative. Then $x \vee y$ is the lowest upper bound of $x$ and $y$, for all $x, y \in H$.

Proof. Let $x<z$ and $y<z$, for some $x, y, z \in H$. By Proposition $4 . \mathrm{L} 2(i v), z \in x \vee z$ and $z \in y \vee z$. Hence $z \in x \vee z \subseteq x \vee(y \vee z)=(x \vee y) \vee z$. Hence there exists $t \in x \vee y$, such that $z \in t \vee z$. Thus by Proposition $4 . \mathrm{I} 2(v), t<z$. Therefore, $x \vee y<z$.

The following example shows that the converse of Theorem 4.15 is not correct, in general.

Example 4.16. In Example 4.7, we can see $(H, \circ)$ is a bounded commutative hyper $K$-algebras.

\begin{tabular}{c|cccc}
$\vee$ & 0 & $a$ & $b$ & 1 \\
\hline 0 & $\{0, a, b, 1\}$ & $\{a, b, 1\}$ & $\{b\}$ & $\{1\}$ \\
$a$ & $\{a, b, 1\}$ & $\{a, b, 1\}$ & $\{a, b, 1\}$ & $\{1\}$ \\
$b$ & $\{b\}$ & $\{a, b, 1\}$ & $\{b, 1\}$ & $\{1\}$ \\
1 & $\{1\}$ & $\{1\}$ & $\{1\}$ & $\{1\}$
\end{tabular}

By easy calculations we get $(H, \circ, 0)$ is a hyper $K$-algebra with condition $(L)$ and for all $x, y \in H$, $x \vee y$ is the lowest upper bound of $x$ and $y$ but $\wedge$ is not an associative operator.

Theorem 4.17. Let $H$ be a hyper $K$-algebra with condition $(L)$. Then for any $x, y \in H$, we have $x \in x \wedge(x \vee y)$ and $x \in x \vee(x \wedge y)$.

Proof. Since $x<x \vee y$, there exists $t \in x \vee y$ such that $x<t$. Hence $x \in x \wedge t$ and so $x \in x \wedge(x \vee y)$. Also, since $x \wedge y<x$, there exists $u \in x \wedge y$ such that $u<x$ and so $x \in x \vee u$. Therefore, $x \in x \vee(x \wedge y)$.

Corollary 4.18. Let $H$ be a hyper $K$-algebra with condition $(L)$ and $\wedge$ be associative. Then $(H, \wedge, \vee)$ is a superlattice.

Proof. By Propositions 4.4(i), (ii), (iv) and 4.52 and by Theorems 4.14 and 4.17 , the proof is obvious.

In the following, we prove that in any hyper $K$-algebra $H$ of order 3 with condition $(L) \wedge$ is associative and so by Corollary $4.18,(H, \wedge, \vee)$ is a superlattice.

Proposition 4.19. Let $H=\{0, a, 1\}$ be a hyper $K$-algebra with condition $(L)$. Then the following hold:

(i) $a^{\prime}=a$,

(ii) $a \wedge a=\{a\}$ or $\{0, a\}$,

(iii) $a \wedge 1=1 \wedge a=\{a\}$ or $\{0, a\}$,

(iv) If $a \wedge 1=\{a\}$, then $1 \wedge 1=\{1\}$.

Proof. (i) By Lemma $4 . \Pi(i), 0^{\prime}=1$ and $1^{\prime}=0$. If $a^{\prime}=1$, then by $\left(L_{3}\right), a=\left(a^{\prime}\right)^{\prime}=1^{\prime}=0$, which is a contradiction. By similar way $a^{\prime} \neq 0$. Thus $a^{\prime}=a$.

(ii), (iii) By Proposition $4.4(i v), 1 \notin a \wedge a$ and $1 \notin a \wedge 1$. Thus $a \wedge a=\{a\}$ or $\{0, a\}$. Also, $a \wedge 1=\{a\}$ or $\{0, a\}$.

(iv) If $a \wedge 1=\{a\}$, then by Proposition 4.4 (vi) and (vii), we consequence $a \circ 1=\{0\}$ and $1 \circ a=\{a\}$.

By Lemma $4 . \Pi(i v), a \circ 0=1 \circ a=\{a\}$ and $0 \circ a=a \circ 1=\{0\}$.

By $(H K 2)$, we have $(a \circ 1) \circ 0=(a \circ 0) \circ 1$ and so $0 \circ 0=a \circ 1=\{0\}$. Therefore, by Lemma $\Pi . \Pi($ iii $), 1 \circ 1=\{0\}$. Hence by Lemma $4 . \Pi(i i), 1 \circ(1 \circ 1)=1 \circ 0=\{1\}$. Also, $1<1$ implies $1 \wedge 1=\{1\}$. 
Theorem 4.20. Let $H=\{0, a, 1\}$ be a hyper $K$-algebra with condition $(L)$. Then $(x \wedge y) \wedge z=$ $x \wedge(y \wedge z)$, for all $x, y, z \in H$.

Proof. By Proposition $4.8(i)$, if at least one of $x, y$ or $z$ are equal to $\{0\}$, then $(x \wedge y) \wedge z=x \wedge(y \wedge z)$. Also, if $x=y=z=a$, then by Proposition $4.8(i i),(x \wedge y) \wedge z=x \wedge(y \wedge z)$. Hence we consider the following cases:

Case 1. Assume $x=y=a, z=1$. Then by Proposition $1.1 \mathrm{~T}($ ii $)$ and (iii), $a \wedge a=\{a\}$ or $\{0, a\}$ and $a \wedge 1=\{a\}$ or $\{0, a\}$. By Proposition $4.8(i i i)$, we obtain $(a \wedge a) \wedge 1=a \wedge(a \wedge 1)$.

Case 2. Let $x=a, y=1, z=a$. Then by commutativity, $(a \wedge 1) \wedge a=a \wedge(1 \wedge a)$.

Case 3. Consider $x=a, y=z=1$. By Propositon $4.1 \mathrm{~d}($ iii $), a \wedge 1=\{a\}$ or $\{0, a\}$.

Let $a \wedge 1=\{a\}$. In this case by Proposition $4 . \mathrm{Ig}(i v)$, we have $1 \wedge 1=\{1\}$. Therefore, $(a \wedge 1) \wedge 1=a \wedge(1 \wedge 1)$. Now, let $a \wedge 1=\{0, a\}$. By Proposition $4.4(i), 1 \in 1 \wedge 1$. Hence by Proposition $4.8($ ii $)$ and $($ iii $),(a \wedge 1) \wedge 1=a \wedge(1 \wedge 1)$.

Case 4. If $x=1, y=z=a$, then by commutativity and Case (1), $(1 \wedge a) \wedge a=1 \wedge(a \wedge a)$.

Case 5. Suppose $x=1, y=a, z=1$. Then by commutativity, $(1 \wedge a) \wedge 1=1 \wedge(a \wedge 1)$.

Case 6. Consider $x=y=1, z=a$. Then by commutativity and Case $(3),(1 \wedge 1) \wedge a=1 \wedge(1 \wedge a)$.

Case 7. Assume $x=y=z=1$. Then the proof is obvious.

Corollary 4.21. Let $H=\{0, a, 1\}$ be a hyper $K$-algebra with condition $(L)$. Then $(H, \wedge, \vee)$ is a superlattice.

Proof. By Corollary 4.18 and Theorem 4.20 , the proof is clear.

In the next example we show that Theorem 4.20 , is not correct for a hyper $K$-algebra of order more than 3 , in general.

Example 4.22. Let $H=\{0, a, b, 1\}$ and the hyperoperation" $\circ "$ on $H$ defined as follows:

\begin{tabular}{c|cccc}
$\circ$ & 0 & $a$ & $b$ & 1 \\
\hline 0 & $\{0, a, b, 1\}$ & $\{0, a, b, 1\}$ & $\{0\}$ & $\{0\}$ \\
$a$ & $\{a, b, 1\}$ & $\{0, a, b, 1\}$ & $\{0, a\}$ & $\{0\}$ \\
$b$ & $\{b\}$ & $\{b\}$ & $\{0, a, b, 1\}$ & $\{0, a, b, 1\}$ \\
1 & $\{1\}$ & $\{b\}$ & $\{a, b, 1\}$ & $\{0, a, b, 1\}$
\end{tabular}

Then $(H, \circ, 0)$ is a hyper $K$-algebra with condition $(L)$. We can see that $\wedge$ is not an associative operator. Because

$$
a \wedge 0=\{0\}, a \wedge a=\{0, a\}, a \wedge b=\{0, a, b\}, a \wedge 1=\{a\}, 1 \wedge 1=\{0, a, b, 1\},
$$

and

$$
a \wedge(1 \wedge 1)=a \wedge\{0, a, b, 1\}=\{0, a, b\} \neq\{a\}=a \wedge 1=(a \wedge 1) \wedge 1
$$

\section{Conclusions}

In this paper, by considering the concepts of hypersemilattice and superlattice, the relation between commutative and positive implicative hyper $K$-algebras with hypersemilattices are studied. Moreover, by adding some conditions the relation between bounded commutative hyper $K$-algebras with superlattices is investigated and it is proved that any hyper $K$-algebra $H$ of order 3 with condition $(L)$ can be a superlattice. 


\section{References}

[1] Z. Bin, X. Ying, H.S. Wei, Hypersemilattices, Chinese Scientific Papers Online, 10 pages, http://www.paper.edu.cn.

[2] R.A. Borzooei, W.A. Dudek, N. Koohestani, On hyper BCC-algebras, Hindawi Publishing Corporation International Journal of Mathematics and Mathematical Sciences, 2006 (2006), $1-18$.

[3] R.A. Borzooei, A. Hasankhani, M.M. Zahedi, Y.B. Jun, On hyper K-algebras, Math Japonica, 1 (2000), 113-121.

[4] X.Z. Guo, X.L. Xin, Hyperlattices, Pure and Applied Mathematics, 20 (2004) 40-43.

[5] Y.B. Jun, M.M. Zahedi, X.L. Xin, R.A. Borzooei, On hyper BCK-algebras, Italian Journal of Pure and Applied Mathematics, 8 (2000), 127-136.

[6] F. Marty, Sur une generalization de la notion degroups, 8th Congress of Mathematics Scandinaves, Stockholm, (1934), 45-49.

[7] J. Mittas, M. Konstantinidou, Sur une nouvelle g'en'eration de la notion de treillis. Les supertreillis et certaines de leurs proprites generales, Annales Sciences Mathématiques Blaise Pascal, Series Mathematics, 25 (1989), 61-83.

[8] T. Roodbari, L. Torkzadeh, M.M. Zahedi, Simple hyper K-algebras, Quasigroups and Related Systems, 16 (2008), 131-140.

[9] X.L. Xin, Hyper BCI-algebras, Discussiones Mathematicae General Algebra and Applications, 26 (2006), 5-19.

[10] M.M. Zahedi, R.A. Borzooei, H. Hasankhani, Some results on hyper K-algebras, Scientiae Mathematicae, 3(1) (2000), 53-59. 